\title{
Wenn die Therapie die Prognose nicht beeinflusst ...
}

\author{
Karl-Ludwig Resch \\ FBK - Deutsches Institut für Gesundheitsforschung, Bad Elster, Deutschland
}

«Die durchschnittliche Lebenserwartung zum Zeitpunkt der Geburt ist in den alten Bundesländern zwischen 1980 und 2002 bei Männern von 69,9 auf 75,6 Jahre und bei Frauen von 76,6 auf 81,3 Jahre gestiegen», war im letzten Jahr im Deutschen Ärzteblatt zu lesen [1]. Illustriert war der Beitrag mit informativen Graphiken, denen zu entnehmen war, gegen welche «Killer-Erkrankungen» sich die moderne Medizin zunehmend erfolgreich zur Wehr setzt. Als da wären z.B. COPD (chronic obstructive pulmonary disease; Gewinn an Lebenserwartung in Jahren: 0,14 bei den Männern und 0,01 bei den Frauen) und Asthma $(0,09$ / 0,06), ischämische Herzkrankheiten $(1,21 / 0,43)$ und zerebrovaskuläre Erkrankungen $(0,75$ / $0,98)$, Magen- $(0,75 / 0,16)$ und Lungenkarzinome $(0,18$ / -0,14 (!)) und Verkehrsunfälle $(0,47 / 0,18)$.

Unklar bleibt bei einer solchen statistischen Betrachtung natürlich, ob die jeweiligen Erkrankungen zunehmend besser therapiert werden können (kurativer Gewinn) oder ob sie weniger häufig auftreten (präventiver Gewinn). Unwahrscheinlich allerdings, dass sich aus der Zunahme der durchschnittlichen Lebenserwartung automatisch ein entsprechender individueller Zugewinn ableiten lässt. Die Lebenserwartung steigt wohl eher, weil mehr Menschen bislang letale Erkrankungen (länger) überleben. Nun ist das aber mit «geretteten Menschenleben» so eine Sache. Einmal gerettet heißt nicht für immer gerettet, sondern vielmehr «at risk» für das nächste Problem.

Dass Erkrankungen das Leben nicht nur bedrohen, sondern es auch einfach weniger lebenswert machen können, dazu präsentiert der Artikel (erwartungsgemäß) keine Daten. Während der Begriff «Mortalität» im oben genannten Artikel nicht weniger als 14-mal auftaucht, findet sich der Begriff «Morbidität» nur ein einziges Mal. «Faktoren wie Ausbildung, Einkommen, Berufsstatus, Mangel an sozialem Rückhalt sowie psychosoziale Belastungen am Arbeitsplatz und in der Familie haben einen wichtigen Einfluss auf Morbidität und
Mortalität», heißt es da ziemlich nichtssagend. Für mich spiegelt dieser Artikel, ohne dass ich den Autoren einen Vorwurf machen kann oder möchte, viel vom aktuellen Dilemma unserer westlichen Mainstream-Medizin wider. Die wird paradigmatisch dominiert vom «add years to life» und mogelt sich um das «add life to years» herum.

«Die dominante Denkfigur des Medizinsystems ist die Akutmedizin, orientiert sowohl auf akutmedizinische Ersterkrankungen wie akutmedizinische Ereignisse auf dem Boden chronischer Krankheitsbilder. Auf diese Akutmedizin konzentrieren sich wissenschaftliche, industrielle und politische Anstrengungen», befand schon vor fast 10 Jahren der damalige Vorsitzende des Sachverständigenrats des Bundesgesundheitsministeriums Friedrich W. Schwartz in einer Analyse [2]. Es ist aber auch zu verlockend in einer Zeit, in der allenthalben «Fakten, Fakten, Fakten» gefordert werden, sich mit Themen zu beschäftigen, in denen dies vorhersehbar möglich ist. Mortalität ist halt einfach ein «harter Endpunkt», nicht zu vergleichen mit visuellen Analogskalen für Schmerz und Befinden oder undurchsichtigen Scores der Lebensqualität, bei denen Hypochonder und Simulanten das Blaue vom Himmel herunter lügen können.

Kein Wunder, dass sich da viele Menschen nicht verstanden fühlen, dass sie nach Alternativen suchen. Kein Wunder auch, dass viele komplementärmedizinische Ansätze gerade hier ihre Stärke wähnen. Das ist beileibe nicht automatisch opportunistisch, sondern zwanglos aus der Tatsache zu erklären, dass sich gerade traditionelle, primär nichtakademische medizinische Ansätze wie die traditionelle chinesische Medizin, das Ayurveda, aber auch die traditionelle europäische Medizin, um nur einige zu nenne, über Zeiten entwickelt haben, in denen das Arsenal der ursachenspezifischen Kausalinterventionen sehr begrenzt war. Kein Wunder also auch, dass an dieser Stelle in jüngster Zeit fast schon gebetsmühlenhaft für eine mehr an den individuellen Bedürfnissen orientierte Sicht der

\begin{tabular}{ll}
\hline KARGER & @ 2007 S. Karger GmbH, Freiburg \\
$\begin{array}{l}\text { Fax +4976145207 14 } \\
\begin{array}{l}\text { E-mail Information@Karger.de } \\
\text { www.karger.com }\end{array}\end{array}$ & $\begin{array}{l}\text { Accessible online at: } \\
\text { www.karger.com/fok }\end{array}$
\end{tabular}

Prof. Dr. med. Karl-Ludwig Resch 
Dinge geworben wird und dabei gegen die «momentan dominierende Variante der evidenzbasierten Medizin» polemisiert [3], die Bedeutung des «menschlichen Faktors» herausgearbeitet [4] und ein stärkerer Fokus auf Gesundheit gefordert wurde [5] und die Bedeutung des Erkenntnisgewinns aus der Praxis von den Herausgebern aktiv propagiert wird [6, 7]. Der Rückenwind wird stärker für eine evidenzbasierte Medizin, die nicht die oberste Priorität in der technisch-methodischen Perfektion von klinischen Studien sieht (interne Validität). Es wurde wiederholt überzeugend dokumentiert, dass dieser Ansatz gerade den quantitativ mit Abstand produktivsten Forschungsbereich, die Arzneimittelforschung, dafür anfällig macht, «sich mit Fragen [zu] beschäftigen, die ... für die aktuelle Versorgung von geringer Relevanz sind» [8]. Evidenz kann natürlich ebenso gut zu anderen Fragen produziert werden, etwa Fragen aus der Patientenperspektive. «Value Based Medicine» nennt sich dieser jüngste Spross der EBM, der in den USA inzwischen auch von der American Medical Association wahr- und offensichtlich ernst genommen wird [9]. Die gewollte Nähe zum «wirklichen Leben», also eine hohe externe Validität, muss dabei gegebenenfalls mit Abstrichen an der internen Validität erkauft werden.

«Value», der Wert oder Nutzen einer Intervention, ist für den Betroffenen eben eine Funktion mehrerer Teilaspekte. Neben dem Wirksamkeitspotential zählen auch die Implementierbarkeit in das tägliche Leben, die negativen Aspekte (z.B. Risiken und/oder Nebenwirkungen) sowie Aufwand und Kosten für die Bewertung.

Gerade bei Erkrankungen, die kurz- oder längerfristig das Leben bedrohen, mag der Therapieerfolg primär vom Knowhow bzw. dem technischen Können des Behandelnden abhängen, z.B. die Therapie maligner Erkrankungen, von Herzinfarkt oder Schlaganfall. Das gleiche gilt grundsätzlich auch für Erkrankungen, bei denen eine irreversible Progression droht, die therapeutisch kontrolliert oder verlangsamt werden kann wie etwa bei Rheuma. Daneben gibt es aber eine große Zahl teilweise ernsthafter Gesundheitsstörungen, bei denen die Therapie die Prognose nicht beeinflusst, man denke nur an den Schnupfen, die Depression, die Migräne und viele andere «Schmerzsyndrome».

Wenn es aber schwierig oder gar unmöglich ist, das Problem $\mathrm{zu}$ «objektivieren», wenn es quoad vitam (respektive prognosem) unerheblich ist; wenn also die Notwendigkeit der Therapie sich nicht eigentlich am «objektivierbaren Bedarf» orientiert, sondern vielmehr an der Nachfrage, also am subjektiven Bedarf, dann sollte sich daran auch die Formulierung von Forschungsfragen orientieren. Dann sollten sich logischerweise daran auch die Kriterien für den Therapieerfolg orientieren. Dann sollte die subjektive Einschätzung seitens des Patienten im Zentrum stehen - und damit automatisch der wahrnehm- bare Effekt («perceived effectiveness») Vorrang vor Fragen der Wirksamkeit («efficacy») haben. Damit ist in direkter Logik die Priorität von Kontrollgruppen mit einer anderen therapeutischen Strategie (bzw. «unbehandelt») verbunden. Placebokontrollen können keine patientenrelevante Information erwarten lassen, die Verblindung würde die «real life situation» verzerren.

Übrigens lässt sich hier zwanglos eine Brücke schlagen zur International Classification of Functioning, Disability and Health (ICF), jener Klassifikation, die 2001 von der WHO verabschiedet und über die sogenannte Reha-Richtlinie des Gemeinsamen Bundesausschusses seit 2004 auch in Deutschland für die Rehabilitation maßgeblich und verbindlich ist [10]. Sie klassifiziert Gesundheitsstörungen im Gegensatz zur bekannteren «Schwesterklassifikation» ICD nicht vor dem Hintergrund von Ätiologie und Pathogenese, sondern beschreibt Funktionen, Strukturen und Aktivitäten, die durch entsprechende Störungen betroffen sind und die Auswirkungen auf die Teilhabe am gesellschaftlichen Leben - alles unter Würdigung von Kontextfaktoren (Umwelt- und persönliche Faktoren).

Analysiert man die Auswirkungen der letzten Gesundheitsreformen in Deutschland (und anderen Ländern) unter den hier angestellten Überlegungen, wird schnell klar, dass gerade Therapien von Gesundheitsstörungen, die typischerweise die Prognose nicht beeinflussen, überproportional aus dem Katalog der Sozialversicherung ausgegliedert wurden. Andererseits scheint mir gerade in diesem Bereich eine besondere Stärke naturheilkundlicher und vieler komplementärmedizinischer Ansätze zu liegen.

\section{Literatur}

1 Weiland SK, Rapp K, Klenk J, Keil U: Zunahme der Lebenserwartung. Größenordnung, Determinanten und Perspektiven. Dt Ärzteblatt 2006;103:A1072-7.

2 Schwartz FW, Bitzer EM, Dörning H, Grobe TG, Krauth Ch, Schlaud M, Schmidt Th, Zielke M: Gutachten. Gesundheitsausgaben für chronische Krankheiten in Deutschland - Krankheitskostenlast und Reduktionspotentiale durch verhaltensbezogene Risikomodifikation. Lengerich, Pabst, 1999.

3 Saller R: Ökonomie und Evidenz. Zur authentischen Evaluation komplementärmedizinischer Ansätze. Forsch Komplementärmed 2006;13:208-9

4 Michalsen A: Die Komplementärmedizin und der «menschliche Faktor». Forsch Komplementärmed 2006;13:68-69.

5 Melchart D: Gesundheitsförderung und Prävention in der Medizin - mehr «befähigen» als «verordnen». Forsch Komplementärmed 2007;14:68-69.

6 Walach H, Michalsen A: Praxis und Erkenntnis. Forsch Komplementärmed 2006;13:4-5

7 Matthiesen PF: Ärztliche Praxis und wissenschaftlicher Status in der Medizin Forsch Komplementärmed 2006;13:136-9.

8 Dieppe P, Chard J, Tallon D, Egger M: Funding clinical research. Lancet 1999; 353:1626.

9 Brown MM, Brown GC: Evidence-based to Value-based Medicine. American Medical Association, 2004.

10 www.who.int/classifications/icf/site/icftemplate.cfm. 